\title{
Formation of Dynamic Topographic Patterns During Electron Beam Induced Etching of Diamond
}

\author{
Aiden A. Martin ${ }^{1}$, Alan Bahm², James Bishop ${ }^{3}$, Igor Aharonovich ${ }^{3}$ and Milos Toth ${ }^{3}$ \\ 1. Lawrence Livermore National Laboratory, Livermore, California, USA. \\ 2. FEI Company, 5350 Northeast Dawson Creek Drive, Hillsboro, Oregon, USA. \\ 3. School of Physics and Advanced Materials, University of Technology, Sydney, 15 Broadway, Ultimo, \\ New South Wales 2007, Australia.
}

Spontaneous formation of complex geometric patterns is an interesting phenomenon that provides fundamental insights into underlying roles of symmetry breaking, anisotropy and non-linear interactions [1]. Here, dynamic, highly ordered topographic patterns on the surface of diamond that span multiple length scales and have a symmetry controlled by the chemical species of precursor gas used in electron beam induced etching (EBIE) are presented [2].

EBIE is a high resolution, direct-write nanofabrication technique in which a precursor gas and an electron beam are used to realize etching [3]. A key advantage of EBIE is the ability to etch materials such as diamond that are resistant to conventional chemical etch processes, without introducing damage to the substrate as observed in ion sputtering techniques. EBIE has therefore recently been used to fabricate components for photonic and electronic applications.

Formation of topographic patterns in diamond was investigated under varying gaseous precursor conditions and crystallographic direction. EBIE experiments were performed at room temperature using an FEI Company Nova NanoSEM environmental scanning electron microscope. Patterns were produced by rastering a $5 \mathrm{keV}, 34 \mathrm{nA}$ electron beam across a region of (001)-oriented or (111)-oriented singlecrystal diamond under a gaseous environment of $\mathrm{H}_{2} \mathrm{O}(9.3 \mathrm{~Pa})$, or a mixture of $\mathrm{H}_{2} \mathrm{O}$ and $\mathrm{NH}_{3}(1.9 \mathrm{~Pa}$ $\mathrm{H}_{2} \mathrm{O}$, $6.4 \mathrm{~Pa} \mathrm{NH}_{3}$ ). Figure 1 shows EBIE performed under these varying conditions which result in drastically different topographic patterns on the diamond surface. The geometries observed during $\mathrm{H}_{2} \mathrm{O}-$ mediated EBIE of (001)- and (111)-oriented diamond is governed primarily by slow etching of (110) planes that are oriented at $90^{\circ}$ with respect to the electron beam axis. On the addition of $\mathrm{NH}_{3}$ slow etching of (111) planes govern the surface geometry.

An anisotropic etch rate kinetics model is proposed to explain the observed patterns, which reveals an electron energy transfer pathway that has been over-looked by existing EBIE theory. In the fundamental modification, the role of energetic electrons is to transfer energy to surface atoms of the solid rather than to surface-adsorbed precursor molecules. This proposal is supported by comparison of the temperature dependent rate of EBIE measured experimentally to the calculated rate using the established EBIE model. Room temperature normalized etch rates differ by up to four orders of magnitude at high temperature $(600 \mathrm{~K})$, indicating that electron induced dissociation of physisorbed precursor molecules is not the dominant reaction mechanism.

The findings of this research can be harnessed to engineer specific surface patterns under various electron beam irradiation environments for controlled wetting, optical structuring and other emerging applications that require nano and micro-scale surface texturing of diamond [4]. 
References:

[1] MC Cross and PC Hohenberg, Rev. Mod. Phys. 65 (1993).

[2] AA Martin et al, Phys. Rev. Lett. 115 (2015).

[3] I Utke, S Moshkalev, and P Russell in "Nanofabrication Using Focused Ion and Electron Beams:

Principles and Applications" (Oxford University Press, New York, 2012).

[4] A portion of this work was funded by FEI Company and the Australian Research Council (Project Number DP140102721). A portion of this work was performed under the auspices of the U.S. DOE by LLNL under Contract DE-AC52-07NA27344. I.A. is the recipient of an Australian Research Council Discovery Early Career Research Award (Project Number DE130100592).
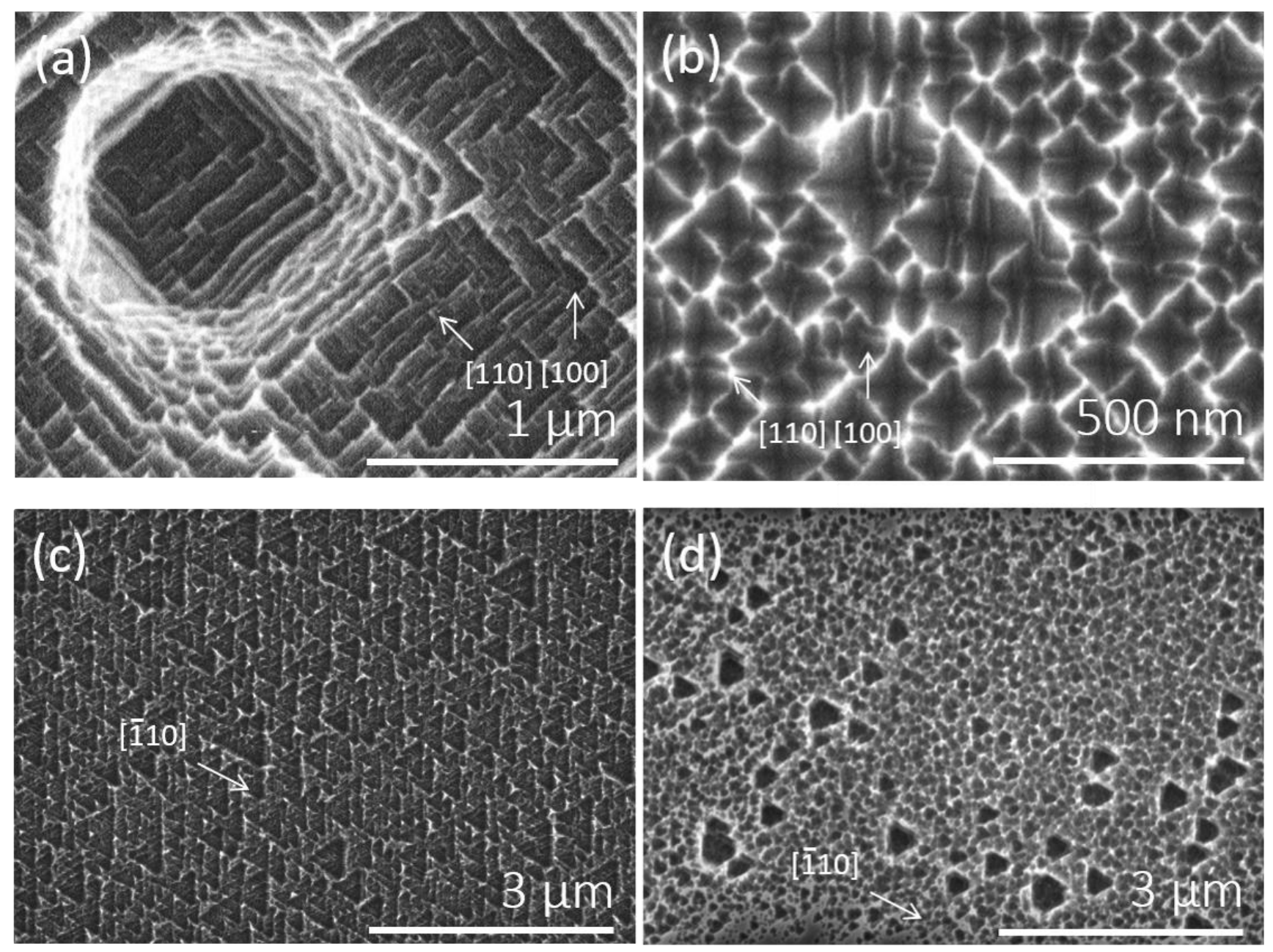

Figure 1. Topographic patterns formed during $\mathrm{H}_{2} \mathrm{O}$-mediated electron beam induced etching of singlecrystal diamond performed using a $5 \mathrm{keV}, 34 \mathrm{nA}$ electron beam. (a) Expanding rhombohedra formed on the surface of (001)-oriented diamond. (b) Inverted pyramids formed on the surface of (001)-oriented diamond with the addition of gaseous $\mathrm{NH}_{3}$. (c) Trigons formed on the surface of (111)-oriented diamond. (d) Trigons formed on the surface of (111)-oriented diamond with the addition of gaseous $\mathrm{NH}_{3}$. 